\title{
Dependence of silkworm productivity indicators on life expectancy of butterflies
}

\author{
$U$ Khudayberideva ${ }^{1,2, *}, S$ Navruzov $^{1,2}, B$ Nasirillaev $^{1,2}, C H$ Bekkamov $^{1,2}, N$ \\ Abdukayumova $^{1,2}$, and $K H$ Fozilova $^{1,2}$ \\ ${ }^{1}$ Tashkent State Agrarian University, University str., 2, Tashkent province, Uzbekistan \\ ${ }^{2}$ Scientific Research Institute of Silk Production, Tashkent, Uzbekistan
}

\begin{abstract}
In this article, the current state of silkworm breeding in Uzbekistan is highlighted by the fact that cocoons, raw silk and silk products grown are not competitive enough. About the role of selection in increasing the volume of cocoon cultivation and improving its quality. This includes ways to influence the genetic characteristics of newly created breeds and their hybrids, which contribute to the improvement of cocoon productivity and technological performance. The importance of population-specific laws, as well as the methodology of experiments, the degree of interdependence of selection traits, experiments in two groups of silkworm breeds and systems, in three gradations, and the results obtained on them. Prospects for the application of experimental results in science and practice are also discussed.
\end{abstract}

\section{Introduction}

In an era of rapidly evolving market economies, competition is constantly increasing, and the cultivation of competitive products can be achieved through the application of scientific innovations in production and the reduction of product costs $[1,2]$. Despite the fact that a lot of work has been done in this direction, unfortunately, the productivity, quality and technological indicators of cocoons grown in our country are still insufficient. This requires the full application of existing scientific developments in production, as well as in-depth research in silkworm breeding and breeding, and the discovery of new genetic potentials for the productivity of individuals [2-4]. Along with the scientific developments in this area, which have been conducted and created to date, we have tried to study the relationship between the life expectancy of silkworm butterflies and their productivity through our chosen topic. In order to grow a rich and high-quality cocoon, it is necessary to pay more attention to selection work and feeding techniques. In this regard, our selection scientists usually work with populations that change from generation to generation as a result of artificial selection and crossbreeding [5,6].

Hence, a good knowledge of the laws inherent in populations can ensure the success of selection work. Population genetics is the study of genetic patterns and phenomena in natural and artificial populations [5, 7]. Because populations are made up of a large number

* Corresponding author: umida.xudayberdieva.91.@, mail.ru 
of individuals (organisms), variability in them can be studied using biometrics or mathematical statistics. Hence, population genetics is shaped by the laws and rules of heredity and biometrics. The use of genetic and biometric methods offers great opportunities for in-depth study of variability in populations of living organisms $[1,8,10]$.

The study of the degree of correlation of the life expectancy of silkworm female butterflies with other selection traits may open up new opportunities for genetics and selection practice $[9,11]$.

The correlation coefficient determines the interdependence of known characters. Based on the correlation coefficient indicator, different characters and properties can be changed in the desired direction by selecting the second character associated with the character to be selected.

The degree of correlation between the selection traits of mulberry silkworm [2-6,10,11] has been studied in detail. In their study, the above-mentioned authors identified a correlation between certain economic value traits and some biochemical indicators.

Nasirillaev B. and Khalilova M. were able to obtain $100 \%$ hybrid seeds without the problem of separating the breeding material into species due to the fact that the father and mother rocks were separated during the ovulation and cocoon period when preparing industrial hybrids. They found that the new hybrid combination, along with germination, is characterized by high performance of the main technological features of silk fiber, which in the future will determine the efficiency of production of this industrial hybrid [9-14].

There is enough starting material to create a new breed and industrial hybrids at the level of demand for mulberry silkworm in terms of productivity and technological characteristics. Further selection of several generations of silkworms of living cocoons on the technological characteristics of selection systems will certainly lead to the creation of new breeds and hybrids of silk fiber at the required level of quality [10-14].

The above-mentioned scientists have proved that the use of synthetic selection measures to raise the breed to a higher level in terms of technological characteristics, which will lead to the production of high quality cocoons in the industry from industrial hybrids "Uzbekistan 5", "Uzbekistan 6", "Golden Valley 2".

\section{Materials and methods}

Our experiments are carried out in the laboratory of "Silkworm Breeding" of the Silk Research Institute. For the first time in our scientific research, an attempt was made to determine the correlation coefficients between the life expectancy of female butterflies and the signs of viability, productivity of their offspring.

For experiments, the Mul silkworm breed Liniya 11 with round cocoon shape, Markhamat breed and Liniya 5, Liniya 66 breed with elongated shape, and Asaka breed were selected.

In the 2018 worm feeding season, Liniyas 11, Liniya 67, Markhamat, Liniya 5, Liniya 66, and Asaka worms were grown in hygrothermal conditions and cocoons were grown. The share of healthy, deaf and dull cocoons was determined from the grown cocoons. Healthy cocoons were then selected and breeding cocoons were selected. Butterflies from the breeding cocoons were crossbred and papilloned on the same day. In order to determine the life expectancy of the butterflies, they were placed in specially insulated bags and allowed to lay eggs in the same container. The butterflies in each container had their own number and it was observed that each butterfly was captured alive at the same time each day (at 9:00 am). This process was carried out until the day the butterfly died and the results were recorded in the experimental notebook. 
Liniya 11, Markhamat, Liniya 5, Liniya 66, and the life expectancy of butterflies of the Asaka breed from the day the butterfly was born until its natural death were counted as the life expectancy of the butterfly.

They were dried for 45 days after all of the experimental butterflies had died. A microscopic examination was then performed to check the health of the butterflies, which was important for the study results. Microscopic analysis was performed based on the requirements of the cellular method of seed preparation, and some disease-detected butterflies were excluded from the experiment.

In our experiments for 2019-2020 were carried out in the worms of the laboratory "Breeding" of the Silk Research Institute. The experimental worms were incubated at a temperature of $24-25{ }^{\circ} \mathrm{C}$ and a relative humidity of $70-75 \%$. After 10 days of incubation, the worms were raised and kept at a temperature of $26-27{ }^{\circ} \mathrm{C}$ in young and $24-25{ }^{\circ} \mathrm{C}$ in adults. When feeding worms with mulberry leaves were cared for at the rate of 1,200 kg of leaves per 1 box. The experimental breeds were fed with mulberry leaves belonging to the Silk Research Institute. The experimental breeds were fed with mulberry leaves belonging to the Silk Research Institute and silkworm cocoons were grown. Cultivated cocoons were collected, cleaned of saliva, and the number of healthy, deaf, and dull cocoons was determined. The cocoons were then sorted and the breeding cocoons were selected. Butterflies from the breeding cocoons were crossbred that day and populated. In order to determine the life expectancy of the female butterflies, the butterflies were placed in special circle-shaped containers and the butterflies were placed in the same container (Fig. 1).
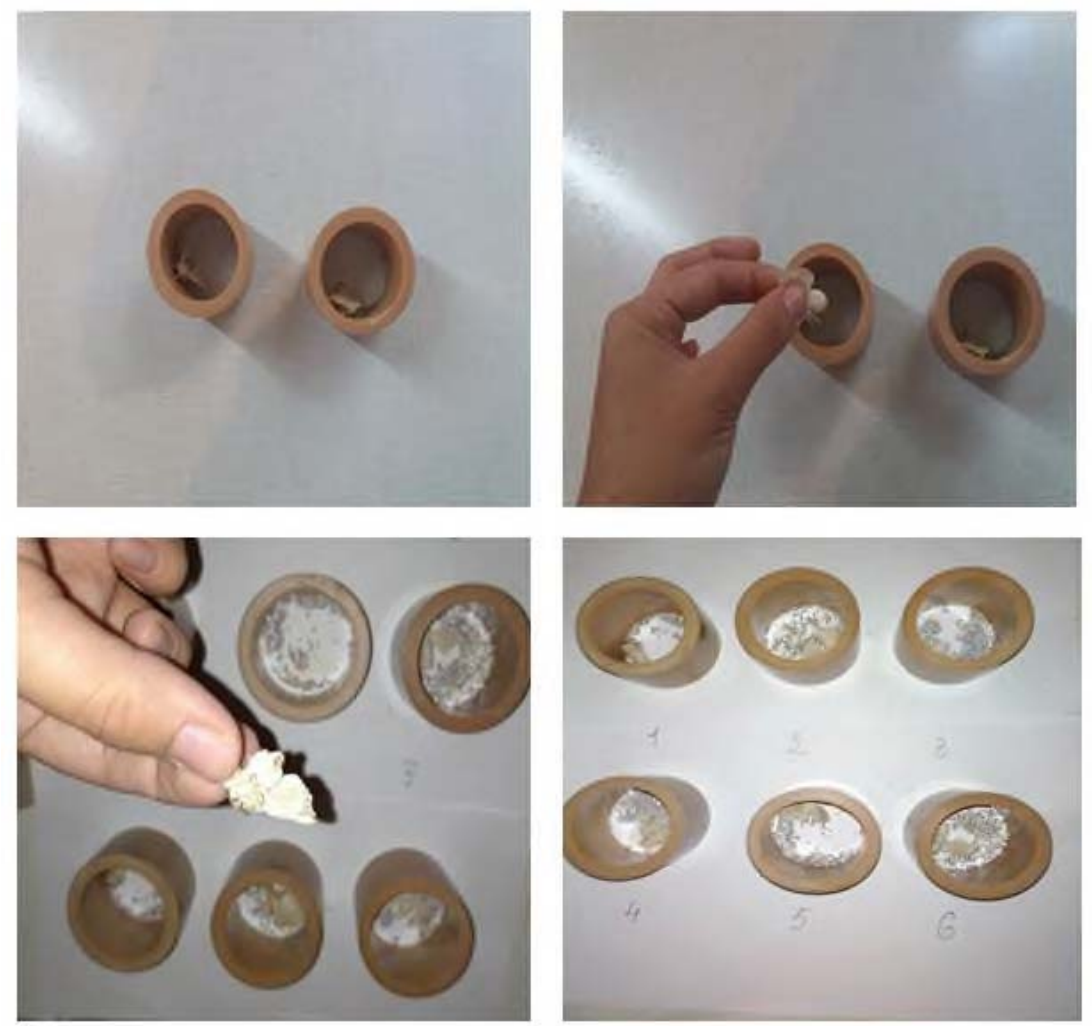

Fig. 1. Butterflies in a circle-shaped container and laying eggs. 
The life expectancy of a butterfly in a species was the number of days the butterfly lived from the day the butterfly was born until the day it died.

Liniya 11, Markhamat, Liniya 5, and Liniya 66 egg laying obtained on the life expectancy of butterflies of the Asaka breed was planned to be divided into 3 gradations according to their life expectancy. To do this, egg yolks were divided into 3 gradations depending on the period in which the butterfly lived.

Round cocoon wrapping Liniya 11, Liniya 67, gradations of Markhamat breeds:

I-gradation: butterflies living 9-12 days;

II-gradation: butterflies living 7-8 days;

III-gradation: butterflies living 1-6 days.

The gradations of Liniya 5, Liniya 66, Asaka strains with elongated cocoons are as follows:

I-gradation: butterflies living 9-11 days;

II-gradation: butterflies living 7-8 days;

III-gradation: butterflies living 1-6 days.

Liniya 11, Markhamat, Liniya 5, Liniya 66, and Asaka breeds were combined according to gradations, egg mixtures were formed and they were revived and cared for.

In order to determine cocoon weight, cocoon shell weight, and silkworm performance, $20 q$ and $20 \hat{\sigma}$ cocoons were randomly selected from each experimental variant and weighed on an electronic scale (Fig. 2). Based on the results obtained, conclusions were drawn about the biological characteristics of the gradations on the life expectancy of female and male butterflies.
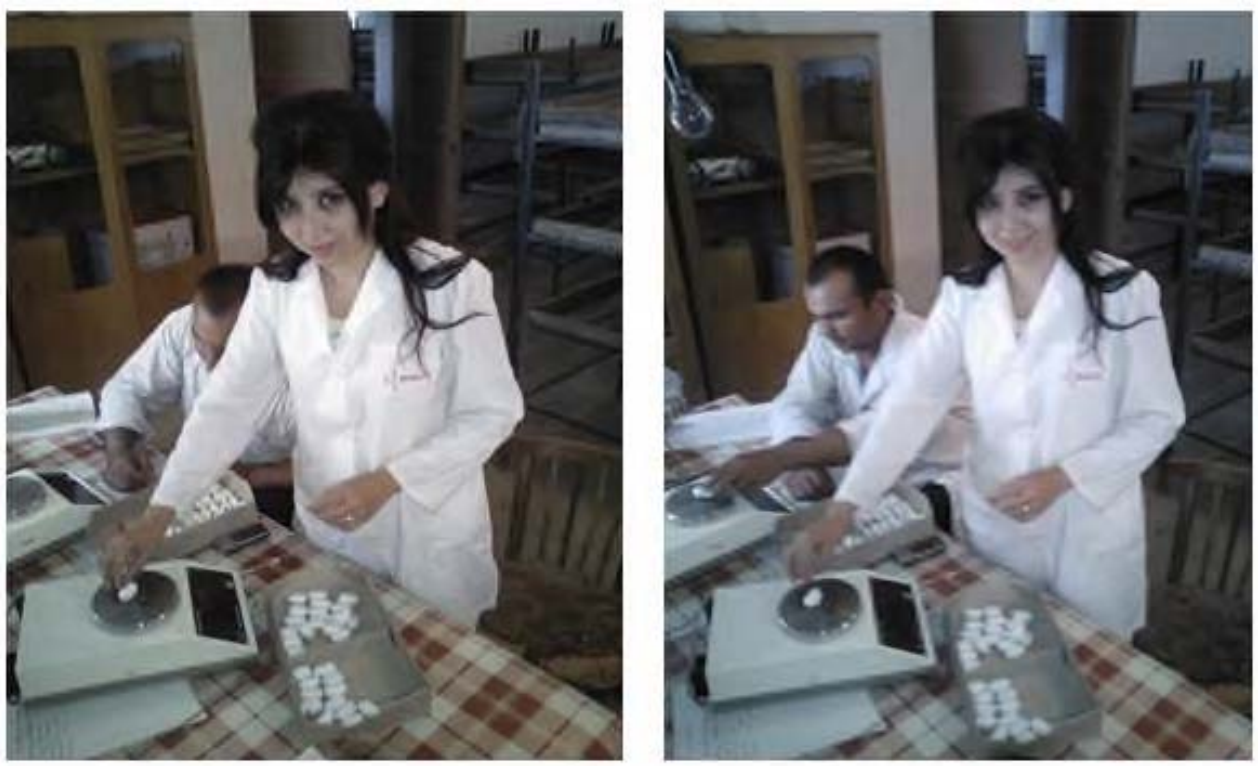

Fig. 2. Silk cocoon weighing process.

\section{Results and discussion}

The effect of the life expectancy of mulberry silkworm butterflies on the productivity of their offspring is shown in Table 1. 
Table 1. Effect of mulberry silkworm butterfly life expectancy on productivity indicators (2018)

\begin{tabular}{|c|c|c|c|c|c|}
\hline Breeds & Gender & $\begin{array}{c}\text { Life expectancy } \\
\text { of butterflies, } \\
\text { days }\end{array}$ & $\begin{array}{c}\text { Silk } \\
\text { cocoon } \\
\text { weight, } g\end{array}$ & $\begin{array}{l}\text { Cocoon shell } \\
\text { weight, } \mathrm{mg}\end{array}$ & $\begin{array}{c}\text { Silk } \\
\text { productivity, \% }\end{array}$ \\
\hline \multirow{3}{*}{ Liniya 5} & 오 & $7.1 \pm 0.408$ & $2.15 \pm 0.013$ & $470 \pm 4.1$ & $21.9 \pm 0.09$ \\
\hline & 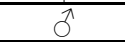 & $8.2 \pm 0.385$ & $1.91 \pm 0.02$ & $497 \pm 2.1$ & $26.0 \pm 0.16$ \\
\hline & Average & $7.65 \pm 0.397$ & $2.03 \pm 0.017$ & $483.5 \pm 3.1$ & $24.0 \pm 0.125$ \\
\hline \multirow{3}{*}{ Liniya 11} & 9 & $8.8 \pm 0.51$ & $1.86 \pm 0.058$ & $408 \pm 4.6$ & $21.9 \pm 0.28$ \\
\hline & $\hat{0}$ & $5.4 \pm 0.50$ & $1.68 \pm 0.011$ & $435 \pm 3.4$ & $25.9 \pm 0.21$ \\
\hline & Average & $7.1 \pm 0.505$ & $1.77 \pm 0.048$ & $421.5 \pm 4$ & $23.9 \pm 0.245$ \\
\hline \multirow{3}{*}{ Liniya 66} & ㅇ & $8.5 \pm 1.02$ & $2.03 \pm 0.422$ & $455 \pm 5.4$ & $22.4 \pm 0.25$ \\
\hline & 0 & $3.9 \pm 0.77$ & $1.65 \pm 0.020$ & $429 \pm 2.23$ & $26.0 \pm 0.26$ \\
\hline & Average & $6.2 \pm 0.895$ & $1.84 \pm 0.221$ & $442 \pm 3.8$ & $24.2 \pm 0.255$ \\
\hline \multirow{3}{*}{ Liniya 67} & 9 & $6.5 \pm 0.67$ & $1.53 \pm 0.038$ & $365 \pm 11.9$ & $23.9 \pm 0.22$ \\
\hline & 0 & $6.0 \pm 0.65$ & $1.28 \pm 0.028$ & $378 \pm 7.8$ & $29.5 \pm 0.34$ \\
\hline & Average & $6.25 \pm 0.66$ & $1.41 \pm 0.033$ & $371.5 \pm 9.85$ & $26.7 \pm 0.28$ \\
\hline \multirow{3}{*}{ Asaka } & q & $9.0 \pm 0.97$ & $2.28 \pm 0.012$ & $431 \pm 1.13$ & $18.9 \pm 0.07$ \\
\hline & $\hat{0}$ & $3.5 \pm 1.29$ & $1.77 \pm 0.05$ & $412 \pm 7.4$ & $23.3 \pm 0.20$ \\
\hline & Average & $6.25 \pm 1.13$ & $2.03 \pm 0.031$ & $421.5 \pm 4.3$ & $21.1 \pm 0.135$ \\
\hline \multirow{3}{*}{ Markhamat } & q & $7.9 \pm 0.85$ & $2.22 \pm 0.02$ & $442 \pm 4.5$ & $19.9 \pm 0.13$ \\
\hline & $\sigma^{\lambda}$ & $5.3 \pm 0.73$ & $1.73 \pm 0.02$ & $424 \pm 2.7$ & $24.5 \pm 0.15$ \\
\hline & Average & $6.6 \pm 0.79$ & $1.98 \pm 0.02$ & $433 \pm 3.6$ & $22.2 \pm 0.14$ \\
\hline
\end{tabular}

If we analyze the figures given in the table above, we will see a repetition of the regularities identified in silkworm breeding up to this time, in other words, we will once again observe that the main traits of mulberry silkworms are directly or inversely correlated with each other.

In particular, the cocoon weight and its silkiness are inversely correlated with the fact that the silkworm cocoon of the Asaka breed in the table weighs $2.28 \mathrm{~g}$, its silkiness is $18.9 \%$, and the female cocoon of the Liniya 5 system is $2.15 \mathrm{~g}$. silkness is 21.9 percent. In contrast, the silkworm cocoon of Liniya 5, which has the longest life expectancy of a butterfly, weighs $1.9 \mathrm{~g}$ and has a silkworm content of $26.0 \%$, while the life expectancy of a female butterfly in Liniya 11 is 8.8 days, compared to that of female worms $1.86 \mathrm{~g}$. Of cocoons and their silkiness is $21.9 \%$, while the figures for males of the same system are 5.4 per day, $1.68 \mathrm{~g}$ and $25.9 \%$, respectively.

The main task of mulberry silkworm breeding and breeding is to increase the number of offspring from the parental forms from year to year. It is known that all the signs of mulberry silkworm with economic value are among the signs of quantity. According to scientists such as N. Dubinin, V.A. Strunnikov, the great representatives of genetics, the transmission of quantitative traits from generation to generation occurs under the influence of polygens. Therefore, in order to increase the performance characteristics of mulberry silkworms or to maintain the level achieved by breeders, selection work should be carried out in the breeding population. In addition to the interaction of the completely new selection traits we are studying with the viability, cocoon productivity traits, it is also of great scientific and practical importance to determine their impact on technological performance. In the gradations of the strains, we identified the indicators of the most basic technological features of the cocoons.

In the 2019 worm-feeding season, 300 of the cocoons of the cultivated species were removed without sorting (cocoons that can be fertilized), the spores were killed by heat and dried in the shade. After drying the cocoon samples to the normal humidity level, they were handed over to the Uzbek Research Institute of Natural Fibers on the basis of a code number to determine their technological performance. 
Technological parameters such as raw silk output, output of silk products, degree of cocoon spinning, total fiber length, continuous spinning length and metric number (thinness) of the fiber were determined (Table 2).

The analysis of the technological indicators in Table 2 shows that the indicators of the characters are increasing in the Liniya 5 breed by gradation and, conversely, are declining in the Asaka breed.

If we compare the performance of each technological character separately for the two breeds, the weight of the dry cocoon in the Asaka breed was $0.488 \mathrm{~g}$ in the I-gradation and $0.462 \mathrm{~g}$ in the II-gradation. The yield of raw silk, which is one of the most important characteristics, was $43.0 \%$ in the I-gradation and $42.6 \%$ in the II-gradation. The same trend is observed in the output of silk products, but the total length of the fiber and the metric number are higher in the II degree. The percentage of cocoon thickening is also higher in grade II than in grade I, i.e., $87.7 \%$ and $87.0 \%$, respectively.

Now, if we analyze the intergradation of the same technological traits in the Liniya 5 breed, we can see that the results of this breed are higher than those of the Asaka breed.

The yield of raw silk from dry cocoons was $41.4 \%$ in the lesser-lived III-gradation population of the Liniya 5 breed, compared to $42.0 \%$ in the longer-lived I-gradation. A similar difference was observed in the output of silk products. The coagulation rates are almost the same in grades I and III, i.e. $85.0 \%$; Found to be around $87.3 \%$ and $95.2 \%$, respectively.

The total length of the fiber was $912 \mathrm{~m}$ in the III-gradation, while the I-gradation index was $1021 \mathrm{~m}$, and the I-gradation results for the fiber metric number were also significantly higher than the III-gradation results $-4,152 \mathrm{~m} / \mathrm{g}$ and $3,656 \mathrm{~m} / \mathrm{g}$.

It was first found that the results of the above-mentioned technological characteristics differed significantly in the background of the life expectancy of female butterflies, especially in two species that differ in cocoon weight. Based on the obtained data, it can be recognized that the technological traits of the offspring obtained from butterflies of the Asaka breed that lived longer (9-11 days) are higher than those of the population that lived relatively less (1-6 days). However, the more closely the butterflies lived in the new breed of Liniya 5 with a medium-sized, elongated shape (very high technological performance), the higher the technological potential of their offspring.

As noted in the literature review, the degree of interdependence of character and traits is indicated by the correlation coefficient. If the correlation coefficient ( $r$ ) has a positive value, it means that the characters under experiment are correlated, if the coefficient is around 0 , there is no correlation, and vice versa, if a negative correlation coefficient is determined, these characters are inversely related. 
Table 2. Technological indicators of breeds by gradation (2018)

\begin{tabular}{|c|c|c|c|c|c|c|c|c|}
\hline 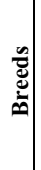 & Gradation & & 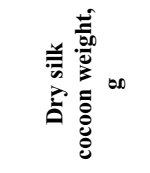 & 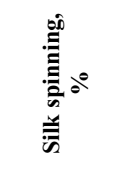 & 禀 ${ }^{\circ}$ & 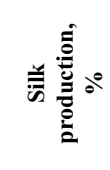 & 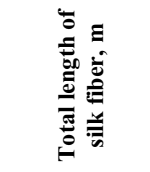 & 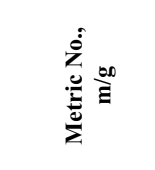 \\
\hline \multirow{8}{*}{ 光 } & \multirow{2}{*}{ I-gradation } & 우 & $0.595 \pm 0.0141$ & $83.6 \pm 1.88$ & $38.5 \pm 1.08$ & $46.0 \pm 0.55$ & $899.4 \pm 47.60$ & $3,940.3 \pm 156.57$ \\
\hline & & $0^{\lambda}$ & $0.582 \pm 0.0118$ & $88.3 \pm 0.93$ & $45.5 \pm 0.58$ & $51.5 \pm 0.33$ & $1,142.6 \pm 1.73$ & $4,363.9 \pm 125.03$ \\
\hline & \multirow{2}{*}{$\begin{array}{c}\text { II - } \\
\text { gradation }\end{array}$} & 우 & $0.622 \pm 0.0150$ & $84.9 \pm 1.52$ & $39.0 \pm 1.07$ & $45.9 \pm 0.80$ & $847.2 \pm 43.24$ & $3,520.9 \pm 165.91$ \\
\hline & & $0^{\lambda}$ & $0.638 \pm 0.0399$ & $89.7 \pm 0.643$ & $47.7 \pm 0.58$ & $53.1 \pm 0.41$ & $1,079.8 \pm 26.47$ & $3,556.8 \pm 73.54$ \\
\hline & \multirow{2}{*}{$\begin{array}{c}\text { III- } \\
\text { gradation }\end{array}$} & q & $0.606 \pm 0.0113$ & $84.0 \pm 1.02$ & $38.2 \pm 0.94$ & $45.4 \pm 0.91$ & $823 \pm 29 . .1$ & $3,578.3 \pm 100.18$ \\
\hline & & $\hat{0}$ & $0.611 \pm 0.0136$ & $86.4 \pm 1.32$ & $44.5 \pm 0.88$ & $52.1 \pm 0.65$ & $1,001.1 \pm 32.14$ & $3,734.5 \pm 210.67$ \\
\hline & \multirow{2}{*}{ Average } & 오 & $0.608 \pm 0.0135$ & $84.2 \pm 1.47$ & $38.6 \pm 1.03$ & $45.8 \pm 0.75$ & $856.5 \pm 39.98$ & $3,679.8 \pm 140.89$ \\
\hline & & $0^{\pi}$ & $0.610 \pm 0.0218$ & $88.1 \pm 0.964$ & $45.9 \pm 0.68$ & $52.2 \pm 0.46$ & $1,074.5 \pm 20.11$ & $3,885.1 \pm 136.41$ \\
\hline \multirow{8}{*}{ 当 } & \multirow{2}{*}{ I-gradation } & $\bar{q}$ & $0.493 \pm 0.0143$ & $87.8 \pm 1.07$ & $40.5 \pm 0.63$ & $46.0 \pm 0.52$ & $773.9 \pm 24.66$ & $3,848.1 \pm 90.83$ \\
\hline & & $0^{\lambda}$ & $0.538 \pm 0.0176$ & $84.3 \pm 2.19$ & $42.5 \pm 1.67$ & $50.0 \pm 1.30$ & $848.5 \pm 46.22$ & $3,875.4 \pm 223.72$ \\
\hline & \multirow{2}{*}{$\begin{array}{c}\text { II - } \\
\text { gradation }\end{array}$} & 우 & $0.517 \pm 0.0141$ & $86.0 \pm 1.53$ & $41.5 \pm 1.13$ & $48.1 \pm 0.68$ & $688.1 \pm 21.53$ & $3,080.3 \pm 144.08$ \\
\hline & & $0^{\pi}$ & $0.519 \pm 0.0127$ & $84.4 \pm 1.94$ & $43.5 \pm 1.56$ & $50.1 \pm 1.14$ & $932.3 \pm 40.81$ & $4,196.9 \pm 133.87$ \\
\hline & \multirow{2}{*}{$\begin{array}{c}\text { III- } \\
\text { gradation }\end{array}$} & 오 & $0.552 \pm 0.0171$ & $87.2 \pm 1.47$ & $40.9 \pm 0.84$ & $46.8 \pm 0.39$ & $765.1 \pm 51.21$ & $3,378.7 \pm 155.90$ \\
\hline & & $0^{\lambda}$ & $0.478 \pm 0.0220$ & $83.5 \pm 2.78$ & $44.6 \pm 1.39$ & $51.4 \pm 0.87$ & $846.9 \pm 36.02$ & $4,018.9 \pm 181.51$ \\
\hline & \multirow{2}{*}{ Average } & 오 & $0.521 \pm 0.0152$ & $87.0 \pm 1.36$ & $41.0 \pm 0.86$ & $47.0 \pm 0.53$ & $742.4 \pm 32.47$ & $3,435.7 \pm 130.27$ \\
\hline & & $\hat{\delta}$ & $0.512 \pm 0.0174$ & $84.1 \pm 2.30$ & $43.5 \pm 1.54$ & $50.5 \pm 1.10$ & $875.9 \pm 41.02$ & $4,030.4 \pm 179.72$ \\
\hline \multirow{4}{*}{ 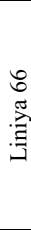 } & I-gradation & +9 and & $0.536 \pm 0.0127$ & $85.9 \pm 1.99$ & $42.8 \pm 1.46$ & $49.7 \pm 0.89$ & $821.8 \pm 29.01$ & $3,618.8 \pm 85.24$ \\
\hline & $\begin{array}{c}\text { II - } \\
\text { gradation }\end{array}$ & $+\underset{0}{a}$ and & $0.588 \pm 0.0152$ & $85.0 \pm 2.47$ & $46.3 \pm 1.47$ & $51.2 \pm 0.94$ & $687.3 \pm 47.25$ & $2,889.9 \pm 208.16$ \\
\hline & $\begin{array}{c}\text { III- } \\
\text { gradation }\end{array}$ & $q$ and & $0.552 \pm 0.0098$ & $89.2 \pm 2.21$ & $45.0 \pm 1.003$ & $48.5 \pm 0.62$ & $787.8 \pm 20.70$ & $3,240.2 \pm 92.63$ \\
\hline & Average & 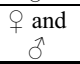 & $0.559 \pm 0.0126$ & $86.7 \pm 2.22$ & $44.7 \pm 1.31$ & $49.8 \pm 0.82$ & $559.3 \pm 32.32$ & $3,249.6 \pm 11.70$ \\
\hline \multirow{4}{*}{$\begin{array}{l}\frac{\pi}{\pi} \\
\frac{\pi}{4}\end{array}$} & I-gradation & $\phi$ and & $0.510 \pm 0.0139$ & $92.1 \pm 0.49$ & $46.6 \pm 0.57$ & $50.6 \pm 0.50$ & $719.2 \pm 32.05$ & $3,007.6 \pm 70.38$ \\
\hline & $\begin{array}{c}\text { II - } \\
\text { gradation }\end{array}$ & +9 and & $0.542 \pm 0.0181$ & $90.1 \pm 1.48$ & $45.0 \pm 1.63$ & $50.1 \pm 2.01$ & $722.2 \pm 27.03$ & $2,997.8 \pm 88.51$ \\
\hline & $\begin{array}{c}\text { III- } \\
\text { gradation }\end{array}$ & $+\underset{i}{i}$ and & $0.547 \pm 0.0156$ & $89.5 \pm 1.24$ & $43.4 \pm 1.18$ & $48.5 \pm 0.95$ & $701.2 \pm 19.92$ & $3,000 \pm 97.44$ \\
\hline & Average & $q$ and & $0.539 \pm 0.0159$ & $90.6 \pm 1.07$ & $45.0 \pm 1.13$ & $49.7 \pm 1.15$ & $714.2 \pm 26.33$ & $3,001.8 \pm 85.44$ \\
\hline \multirow{3}{*}{ 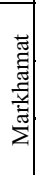 } & I-gradation & $+\underset{i}{i}$ and & $0.488 \pm 0.0147$ & $87.0 \pm 1.16$ & $43.0 \pm 0.89$ & $49.5 \pm 0.84$ & $647.2 \pm 23.59$ & $3,108.8 \pm 73.65$ \\
\hline & $\begin{array}{c}\text { II - } \\
\text { gradation }\end{array}$ & i $\underset{0}{\text { and }}$ & $0.462 \pm 0.0153$ & $87.7 \pm 1.38$ & $42.6 \pm 1.26$ & $48.3 \pm 0.43$ & $729.2 \pm 23.29$ & $3,782.5 \pm 117.95$ \\
\hline & Average & $\phi$ and & $0.475 \pm 0.015$ & $87.4 \pm 1.27$ & $42.8 \pm 1.08$ & $48.9 \pm 0.64$ & $688.2 \pm 23.44$ & $3,445.7 \pm 95.8$ \\
\hline
\end{tabular}

\section{Conclusions}

Based on the above data, it can be concluded that female butterflies of all breeds except the Liniya 5 system lived longer than males in the 4 systems and 2 strains studied in the first place. It is also observed that the longer the butterflies live, the lower the silkiness of the cocoons obtained from them. When the life expectancy of butterflies was compared by breed and system, it was found that sex-marked systems lived longer than other species. However, the fact that cocoon productivity depends primarily on the genotype of the breed and system has been proven in our study.

At the same time, a deeper study of the level of correlation of life expectancy of female silkworm butterflies with other selection traits can create new opportunities for genetics and selection practice, as these results will be useful in further development of silkworm breeding in the current competitive environment. 
It is recommended to monitor the life expectancy of female butterflies in order to improve the viability and cocoon productivity of the breeding material.

\section{References}

1. N. A. Akhmedov, Voris, 21-52 (2014) (in Uzbek)

2. N. Akhmedov, U. Daniyarov, Davr, 57-72 (2014) (in Russian)

3. CH. Bekkamov, N. Akhmedov, S. Valiev, U. Jumanova, KH. Rakhmanova, Zooveterinary, 9, 36-37 (2010)

4. X. Ruiz, M. Almanza, Chilean journal of agricultural research, 78(4), 569-579 (2018)

5. J. Doreswami, S. Gopal, H. Hagedorn, Journal of insect science, 12(1), 21 (2012)

6. KH. Rakhmanova, N. Akhmedov, Zooveterinary, 5, 37-38 (2011)

7. E. Talebi, G. Subramanya, S. Bakkappa, Journal of Agricultural Biological Science, 5, 52-55 (2010)

8. N. Akhmedov, KH. Rakhmanova, Zooveterinary, 3, 14-17 (2013)

9. B. Nasirillaev, M. Jumaniyozov, Zooveterinary, 1(62), 44 (2013) (in Russian)

10. M. Khalilova, B. Nasirillaev, Zooveterinary, 11(120), 36-38 (2011)

11. S. Lezhenko, K. Giyosova, M. Jumaniyozov, B. Nasirillaev, Zooveterinary, 9, 35-37 (2013)

12. SH. Umarov, K. Giyosova, B. Nasirillaev, M. Jumaniyozov, Zooveterinary, 3, 40-41 (2014)

13. S. Navruzov, B. Nasirillaev, N. Rajabov, U. Khudayberdieva, Theoretical \& Applied Science, 144-148 (2020)

14. S. Navruzov, N. Rajabov, U. Khudayberdiyeva, International Journal of Research Culture Society, 4(2), 9-11 (2020) 